\title{
Time to surgery in thoracic cancers and prioritization during COVID-19: a systematic review
}

\author{
Scott C. Fligor, Savas T. Tsikis, Sophie Wang, Ana Sofia Ore, Benjamin G. Allar, Ashlyn E. Whitlock, \\ Rodrigo Calvillo-Ortiz, Kevin Arndt, Mark P. Callery, Sidhu P. Gangadharan \\ Department of Surgery, Beth Israel Deaconess Medical Center, Harvard Medical School, Boston, MA, USA \\ Contributions: (I) Conception and design: SC Fligor, SP Gangadharan; (II) Administrative support: None; (III) Provision of study materials or \\ patients: None; (IV) Collection and assembly of data: SC Fligor, ST Tsikis, AS Ore, K Arndt; (V) Data analysis and interpretation: All authors; (VI) \\ Manuscript writing: All authors; (VII) Final approval of manuscript: All authors. \\ Correspondence to: Sidhu P. Gangadharan, MD, MHCM, FACS. Chief, Division of Thoracic Surgery and Interventional Pulmonology, Beth Israel \\ Deaconess Medical Center, 185 Pilgrim Road, Boston, MA 02215, USA. Email: sgangadh@bidmc.harvard.edu.
}

Background: Coronavirus disease 2019 (COVID-19) has overwhelmed hospital resources worldwide, requiring widespread cancellation of non-emergency operations, including lung and esophageal cancer operations. In the United States, while hospitals begin to increase surgical volume and tackle the backlog of cases, the specter of a "second wave," with a potential vaccine months to years away, highlights the ongoing need to triage cases based upon the risk of surgical delay. We synthesize the available literature on time to surgery and its impact on outcomes along with a critical appraisal of the released triage guidelines in the United States.

Methods: We performed a systematic literature review using PubMed according to preferred reporting items for systematic reviews and meta-analyses guidelines evaluating relevant literature from the past 15 years.

Results: Out of 679 screened abstracts, 12 studies investigating time to surgery in lung cancer were included. In stage I-II lung cancer, delayed resection beyond 6 to 8 weeks is consistently associated with lower survival. No identified evidence justifies a $2 \mathrm{~cm}$ cutoff for immediate versus delayed surgery. For stage IIIa lung cancer, time to surgery greater than 6 weeks after neoadjuvant therapy is similarly associated with worse survival. For esophageal cancer, 254 abstracts were screened and 23 studies were included. Minimal literature addresses primary esophagectomy, but time to surgery over 8 weeks is associated with lower survival. In the neoadjuvant setting, longer time to surgery is associated with increased pathologic complete response, but also decreased survival. The optimal window for esophagectomy following neoadjuvant therapy is 6 to 8 weeks.

Conclusions: In the setting of the COVID-19 pandemic, timely resection of lung and esophageal cancer should be prioritized whenever possible based upon local resources and disease-burden.

Keywords: Time to surgery; lung cancer; esophageal cancer; surgical delay; cancer outcomes

Submitted Jul 09, 2020. Accepted for publication Sep 30, 2020.

doi: $10.21037 /$ jtd-20-2400

View this article at: http://dx.doi.org/10.21037/jtd-20-2400

\section{Introduction}

Coronavirus disease 2019 (COVID-19) continues to strain global health systems with exponential spread and high morbidity. Parts of the United States were overwhelmed with the need to ration ventilators and other hospital resources. In order to maximize hospital capacity, the Centers for Disease Control and Prevention released interim guidance in March 2020 recommending rescheduling of elective surgical procedures and moving elective urgent procedures to the outpatient setting when 
possible (1). The American College of Surgeons (ACS) subsequently echoed these recommendations and published specialty-specific guidelines to triage elective surgical cases in accordance with local conditions (2). As COVID-19 cases continue to escalate in the United States with over two million cases and one-hundred thousand deaths as of June 2020, elective surgical volume has plummeted-including for cancer operations. While states have begun to reopen and resume elective procedures, a large backlog of surgical cases requires ongoing prioritization and an impending "second wave" will continue to constrain surgical capacity.

For many forms of cancer, surgical resection remains the cornerstone and often first step of curative therapy. Delayed resection may result in further tumor growth, invasion, and metastasis. However, the effects of time to surgery for many cancers have not been well characterized and the "acceptable" wait time prior to worsened outcomes is unclear. Beyond oncologic outcomes, the formidable combination of a cancer diagnosis with delayed surgery creates immense mental and physical challenges for our patients. In the setting of unprecedented hospital strain expected to continue for at least months combined with the glut of accumulated cases needing to be scheduled, it is critical to understand which operations should be prioritized and which can be delayed with minimal risk. The Thoracic Surgery Outcomes Research Network published detailed guidance for lung and esophageal cancer that takes into account hospital resources, urgency of procedure, and potential alternative management strategies. Phase I comprises largely maintained hospital resources and minimal burden from COVID-19, while phases II and III represent significant burden and all surgical procedures are delayed except for conditions threatening survival within days or hours, respectively (3). No previous systematic review has evaluated the impact of time to surgery in lung cancer or in primarily-resected esophageal cancer.

We seek to synthesize the available data on time to surgery and its impact on outcomes in adult patients, in accordance with the Preferred Reporting Items for Systemic Reviews and Meta-analyses (PRISMA) checklist, providing an evidence-based approach to prioritization of lung and esophageal cancer surgery during the COVID-19 pandemic, along with a critical appraisal of the released guidelines.

\section{Methods}

\section{Identification of studies}

This systematic review follows the PRISMA checklist (available at http://dx.doi.org/10.21037/jtd-20-2400) (4). We identified studies that evaluated time to surgery with oncologic outcomes. The PubMed database was searched from January 1, 2005 to March 23, 2020 according to the predefined search strategy; specific search terms are below: ("lobectomy" OR "lung resection" OR "pulmonary resection") AND ("delay" OR "time to surgery" OR "timeto-surgery" OR "timing" OR "time to treatment" OR "time-to-treatment")) OR ("lung cancer" AND "surgery" AND ("time to surgery" OR "time-to-surgery" OR "timing” OR "delay”)); ((“esophagectomy” OR "esophageal resection”) AND ("delay" OR "time to surgery" OR "timeto-surgery" OR "timing" OR "time to treatment" OR "time-to-treatment")) OR ("esophageal cancer" AND "surgery" AND ("Time to surgery" OR "time-to-surgery" OR “timing" OR "delay")).

For each cancer, two authors independently and in duplicate conducted an electronic literature search, screening of eligible records, review of potentially relevant complete articles, and evaluation for inclusion. The reference lists of all included studies were hand-searched for additionally potentially relevant articles.

\section{Study inclusion and data extraction}

Studies were eligible for inclusion if they evaluated the effect of time to surgery on pathologic upstaging or response, disease-free survival, or overall survival. Studies were excluded if they did not separate patients who received surgical treatment from other treatments, if they were not written in English, or if they included patients under 18. The following data were extracted using a predefined extraction form: first author, publication year, study design, number of patients, patient population, neoadjuvant therapy, age, matching/multivariate analysis, outcome measure, time to surgery groups, length of follow up, and summary findings. When given, odds ratios or hazard ratios were extracted.

\section{Assessment of quality and bias}

Levels of evidence were assigned according to the Oxford Centre for Evidence-Based Medicine (5). We utilized the Newcastle-Ottawa Scale to evaluate potential bias for observational studies. Ranging from zero to nine, the scale evaluates patient selection, comparability of patient populations, and outcome assessment (6). Both the level of evidence and the Newcastle-Ottawa Scale were assessed 


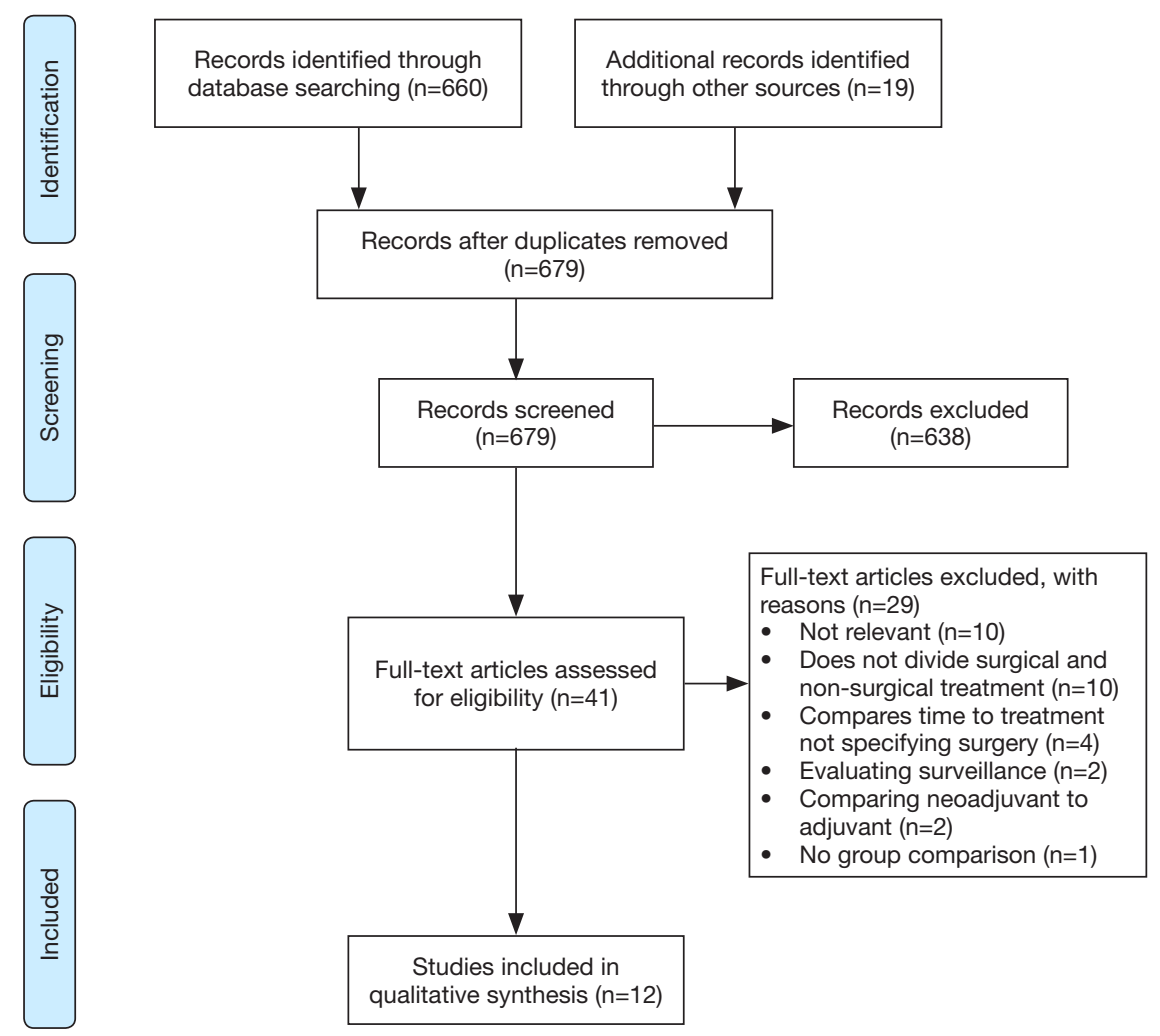

Figure 1 Inclusion flow diagram for time to surgery in lung cancer.

independently by two reviewers and disagreements were resolved through discussion.

\section{Results}

\section{Lung cancer}

A total of 679 abstracts were identified from the search strategy, with 660 from PubMed and an additional 19 abstracts identified through reference list review. A total of 41 full text articles were evaluated and 12 papers met the criteria to evaluate whether surgical delay affects outcomes in lung cancer (Figure 1). Extracted data for included studies are found in Table 1. Included studies predominantly involved early (stage I-II) non-small cell lung cancer (NSCLC), with two papers including all stages of disease and two specifically focusing on Stage IIIa. Nine studies evaluated primary resection, while three papers focused on surgery after neoadjuvant chemoradiotherapy. Most studies evaluated overall survival, two investigated overall survival and upstaging, and four only studied upstaging. All studies were retrospective cohort studies, with a combination of national database use and single-institution experiences. Most studies evaluated time to surgery greater than 4 to 8 weeks, with four studies evaluating a period greater than three months.

Three large studies evaluated stage I NSCLC utilizing the National Cancer Database (NCDB), demonstrating increased upstaging and worse survival with increasing time to surgery $(7,15,17)$. Bott et al. found that time to surgery greater than 8 weeks was associated with pathologic upstaging (OR 1.10, 95\% CI: 1.03-1.16) (7). Samson et al. evaluated the same population with propensity matching, demonstrating that surgical delay over 8 weeks is associated with pathologic upstaging $(\mathrm{P}=0.002)$ although the absolute difference was small (pathologic stage over $1,16.6 \%$ vs. $18.3 \%, \mathrm{P}=0.002)$. Further, they found that surgical delay was associated with increased 30 -day mortality $(2.4 \%$ vs. $2.9 \%, \mathrm{P}=0.01)$ and decreased overall survival (69.2 vs. 57.7 months). Finally, Yang et al. specifically evaluated stage Ia patients finding a time to surgery greater than 38 days was associated with decreased five-year survival (HR 1.13, 


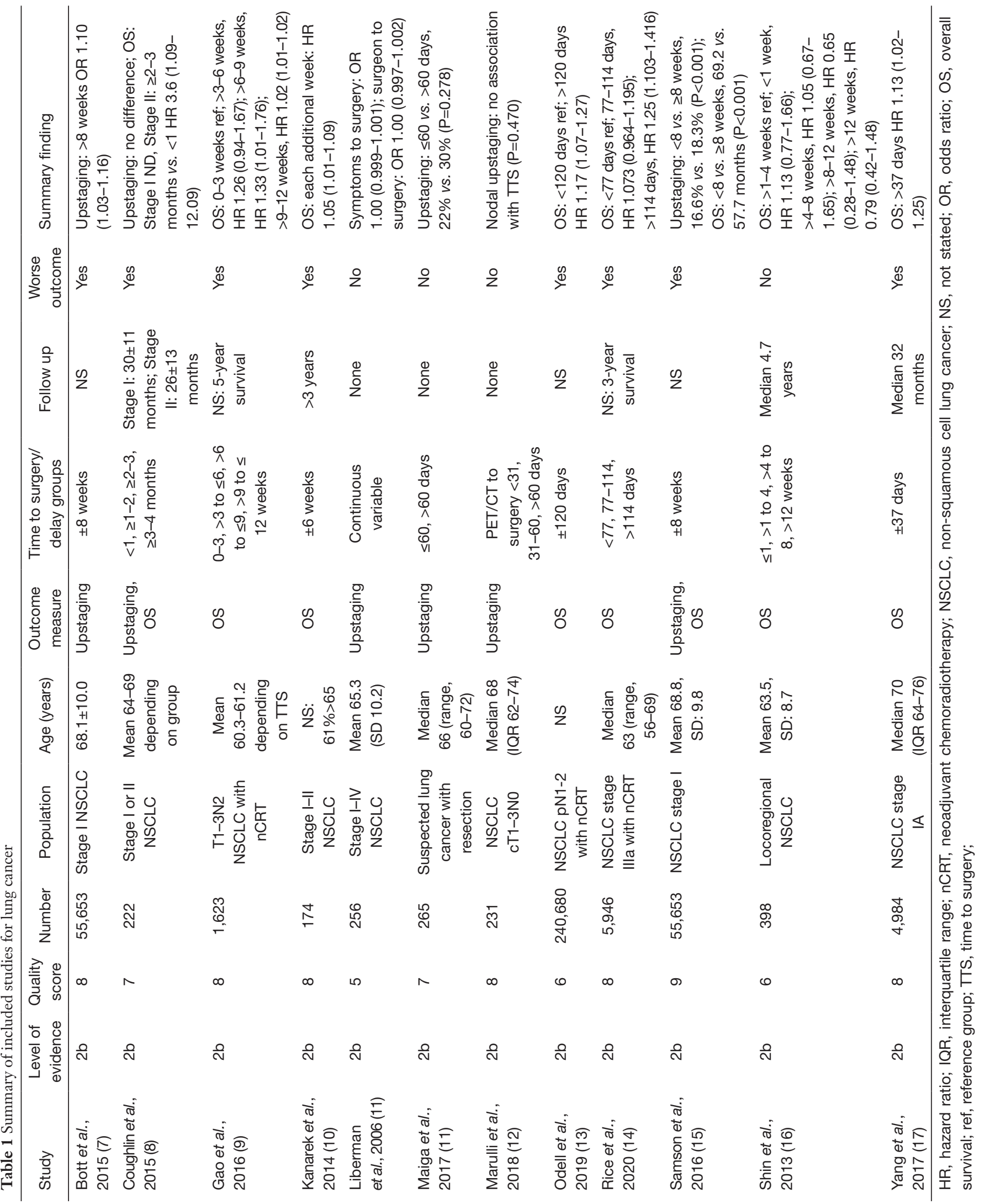


95\% CI: 1.02-1.25) (17).

Several single institution studies also evaluated early stage lung cancer. Coughlin et al. found in patients with stage I lung cancer, time to surgery up to four months was not associated with upstaging or worse survival, but in patients with stage II lung cancer $(n=42)$ there was increased upstaging and worse survival for time to surgery over 2 months. However, this was an unadjusted analysis with very broad confidence intervals (mortality HR: 3.6, 95\% CI: 1.09-12.09) (8). Kanarek et al. had similar findings with each additional week to surgery increasing mortality (additional week HR: 1.04, 95\% CI: 1.00-1.09), although a delay greater than the predefined six-week cut off did not reach significance (10).

For stage IIIa disease, several studies used the $\mathrm{NCDB}$ and found that increased time to surgery after neoadjuvant therapy worsens survival. Gao et al. evaluated 1,623 patients and subdivided time to surgery after neoadjuvant chemoradiotherapy into 3 -week intervals. Compared to the immediate surgery group ( 0 to 3 weeks), time to surgery of 6 to 9 weeks was associated with worse overall 5 -year survival (30.2\% vs. 19.6\%, HR 1.45, 95\% CI: 1.04-2.01). The three to 6-week group trended towards worse overall survival, but this was not significant $30.2 \%$ vs. 22.6\%, HR 1.26, 95\% CI: 0.94-1.67) (9). Rice et al. divided 5,946 patients into three groups divided by the interquartile range, with a short delay group under 77 days and a long delay group over 114 days after neoadjuvant therapy. Notably, they did not exclude patients who underwent chemotherapy or radiotherapy alone, and more patients in the mid and long delay groups had chemotherapy alone. They found decreased three-year overall survival in patients that surgery was delayed over 114 days compared to patients that underwent surgery within 77 days after neoadjuvant chemoradiotherapy after multivariate adjustment (short $59 \%$ vs. long 52\%, HR 1.25, P=0.0005) (14). Finally, Odell et al. investigated compliance with Commission on Cancer lung quality measures, finding that delaying surgery for over 120 days is associated with worse survival (HR 1.17, 95\% CI: 1.07-1.27) (13).

Many of the included studies do not specify the maximum length of delay which may impact the outcomes of their studies if patients with very long delays are included. Furthermore, very few papers discuss the reason for delay given inherent limitations in database studies. Of studies that found a worse outcome with surgical delay, those that clearly discussed the length of delay include the following. In Coughlin et al., all of the patients included had surgery within four months for stage I disease, and within three months for stage II disease. For patients with stage I disease, $77 \%$ of those with a delay of 3 to 4 months were delayed due to OR availability (8). In Gao et al., delays longer than 12 weeks were not included (9). Kanarek et al. reported the upper bound of the $95 \%$ CI for referral to surgery as 74 days (10). Samson et al. did not provide a maximum delay, but provided the interquartile range for the longer delay group as 64-102 days (15). Similarly, Yang et al. stated that $95 \%$ of resections occurred within 4 months of diagnosis (17).

\section{Esophageal cancer}

In total, 254 abstracts were identified from the search strategy, with 248 from PubMed and an additional six abstracts identified through reference list review. Twentyseven full papers were reviewed and ultimately 23 papers met the criteria to evaluate whether surgical delay affects outcomes in esophageal cancer (Figure 2). Extracted data for included studies are found in Table 2. Only four studies evaluated primary esophagectomy with the remainder evaluating surgery after neoadjuvant therapy. All included studies were retrospective cohort studies with one utilizing propensity matching. Several included studies involved multiple centers and many utilized national databases.

Four articles examined time to surgery in primary esophagectomy, with only one study reaching significance. Raman et al. used the NCDB to evaluate patients with cT1N0M0 esophageal adenocarcinoma (28). There was no association between time to surgery quartiles and overall survival; however, in continuous modeling time under 50 days was associated with improved survival (HR 0.991, 95\% CI: 0.984-0.997) and time to surgery over 100 days was associated with worse survival (HR 1.003, 95\% CI: 1.001-1.006) (28). Visser et al. (in 2017) utilized the Netherlands Cancer Registry. In the surgery-only subgroup, time to surgery greater than 8 weeks was not significantly associated with pathologic upstaging or worse survival (HR: 1.00, 95\% CI: 0.82-1.23) (39). Two small institutional studies were also included, with Visser et al. (2016) being the higher quality study. This study suggested worse overall survival with each additional week of delay, also not reaching significance (HR 1.06, 95\% CI: 0.99-1.13) (38).

The remainder of the studies investigated time to surgery after neoadjuvant therapy. Only four studies found an association between surgical delay and worse outcome. Three of these were large studies utilizing the 


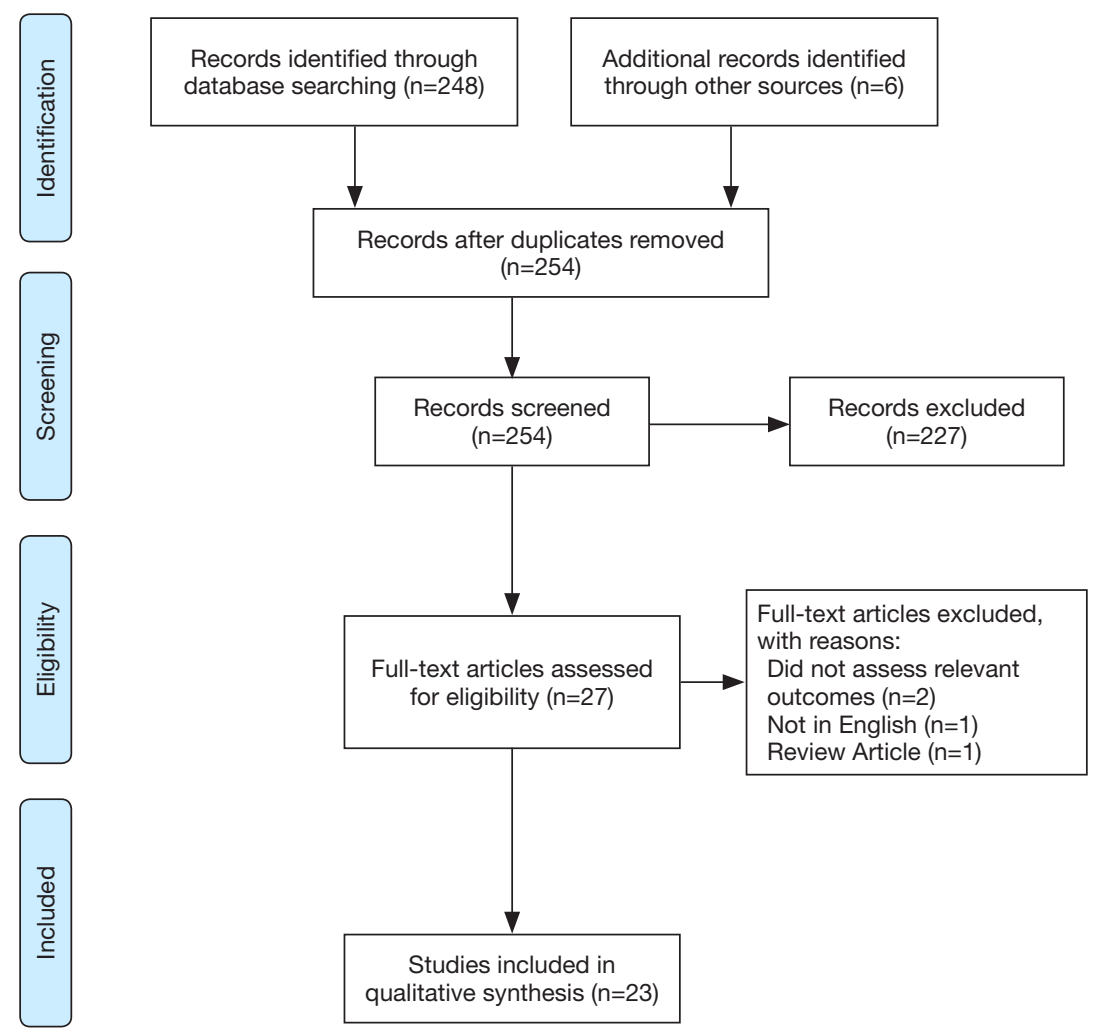

Figure 2 Inclusion flow diagram for time to surgery in esophageal cancer.

NCDB. Ranney et al. evaluated patients with stage II or III esophageal adenocarcinoma who underwent neoadjuvant chemoradiotherapy followed by surgery before or after 56 days. While patients in the over 56-day group had greater pathologic downstaging (OR $1.38,95 \%$ CI: $1.02-1.85)$, they also demonstrated worse overall survival (HR 1.44, 95\% CI: 1.22-1.71) (29). Lee et al. also used the NCDB but included both adenocarcinoma and squamous cell carcinoma, dividing patients into four-time intervals (40 days or less, 41-50, 51-63, >64). Similarly, a time interval greater than 64 days was associated with greater pathologic complete response (OR 1.53, 95\% CI: $1.19-1.98$ ) but worse overall survival (HR 1.16, 95\% CI: 1.01-1.33) (27). Franko et al. (in 2016) evaluated adenocarcinoma and found that time to surgery over 9 weeks was associated with decreased survival (HR 1.19, 95\% CI: 1.03-1.38) (19). The remaining study was Wang et al., who using the Taiwan Cancer Database found that time to surgery over 90 days was associated with worse overall survival, compared to 30-59 days (HR 2.01, 95\% CI: 1.14-3.54) (40). Of these studies demonstrating worsened survival with surgical delay, the maximum length of delay was provided in all of them.
Ranney et al. excluded patients with esophagectomy over 90 days from nCRT (29). Lee et al. had a maximum delay of 210 days, but importantly demonstrated worsened survival in the 41-50 and 51-63 days subgroups compared to the reference of 40 days or less-negating the impact of extreme delays on survival (27). Franko et al. excluded patients with surgery after 26 weeks (19). Similarly, Wang et al. excluded patients with delay over 180 days (40).

The remaining articles did not find an association between surgical delay and worse outcomes in patients receiving neoadjuvant chemotherapy. Of the thirteen studies evaluating pathologic complete response, five showed improvement with delayed resection ranging from 45 days to 13 weeks $(23,27,31,32,36)$. van der Werf et al. was a well-designed multicenter retrospective cohort study that included 3,102 patients. They differentiated between esophageal adenocarcinoma and squamous cell carcinoma and examined intervals from under 5 weeks to over 15 weeks. For patients with adenocarcinoma and squamous cell carcinoma, an interval of more than 10 weeks and more than 13 weeks respectively was associated with a higher probability of pathologic complete response. Unfortunately, 







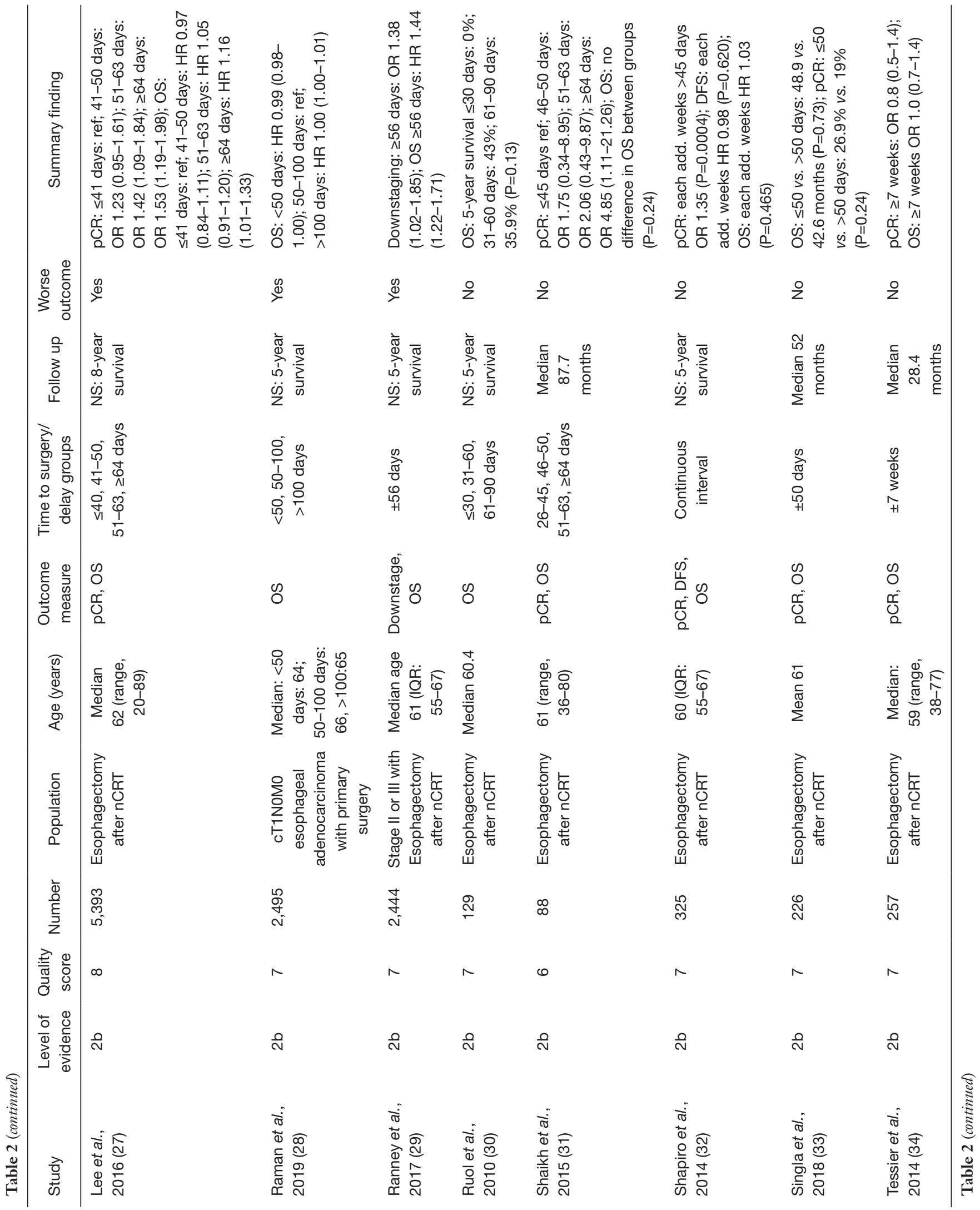




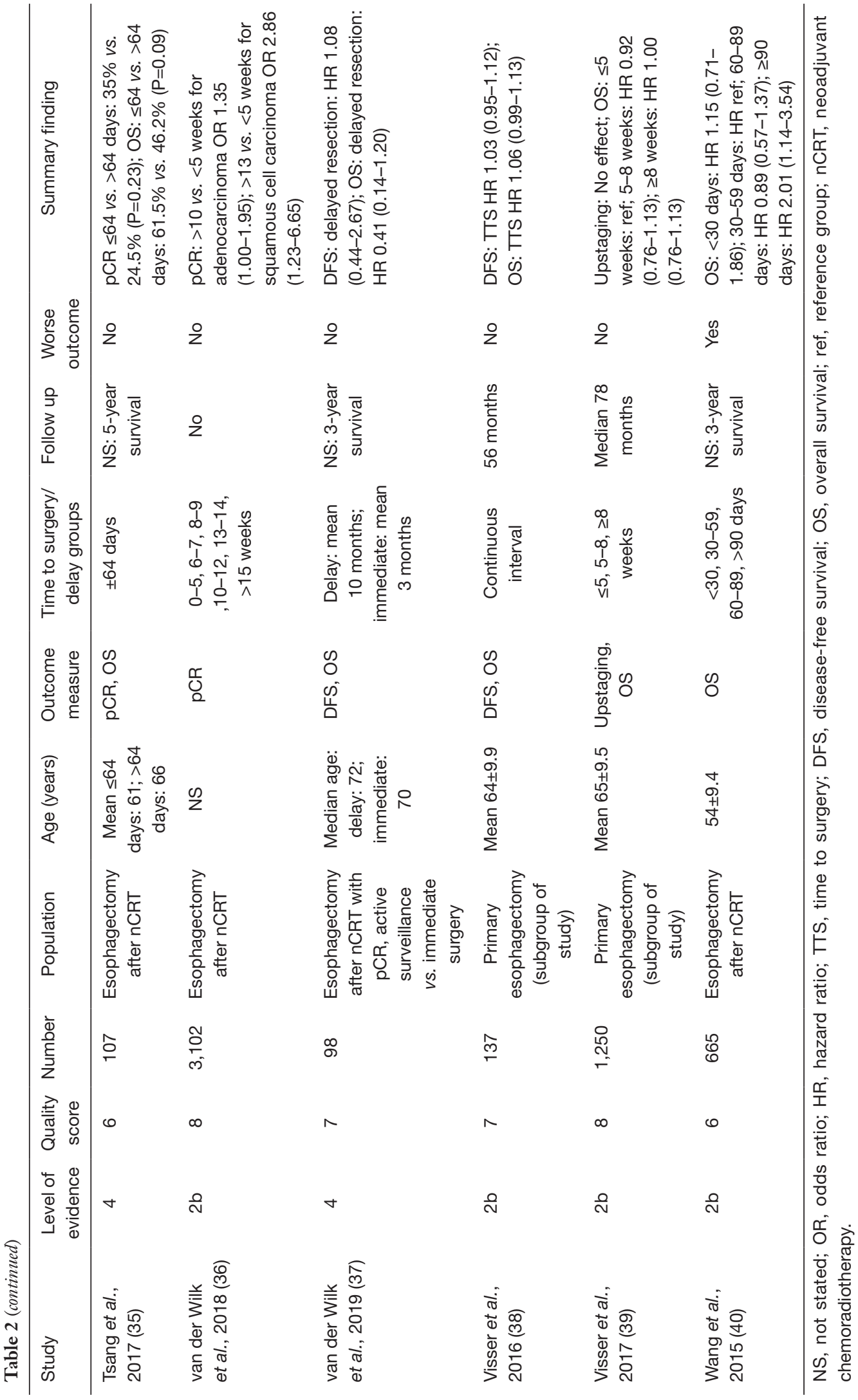


they did not examine overall survival or disease-free survival (36). Franko et al. (in 2018) investigated squamous cell carcinoma in the $\mathrm{NCDB}$ with time to surgery greater than 9 weeks not associated with worse survival (20). Klevebro et al. utilized the Swedish National Register for Esophageal and Gastric Cancer, evaluating patients with time to surgery greater than or less than 49 days. There was no difference in histological response or on overall survival (HR 0.99, 95\% CI: 0.79-1.24) (26).

\section{Discussion}

\section{Lung cancer}

There are no clear guidelines for surgical timing in lung cancer. The National Comprehensive Cancer Network (NCCN) recommends not delaying surgical resection beyond 60 days following completion of clinical staging to prevent upstaging, but does not specify a time from diagnosis to surgery (41). The ACS Commission on Cancer recommends surgery after neoadjuvant chemotherapy occur within 120 days (42). Guidelines for triage of lung cancer surgery during the COVID-19 pandemic highlight different "phases" based upon hospital resources and COVID-19 prevalence. In "Phase I" when capacity is largely preserved, immediate surgery is recommended for solid or predominantly solid lung cancers over $2 \mathrm{~cm}$, node positive cancers, and cancer post neoadjuvant therapy. This is based upon the evaluation of whether survival is "likely to be compromised by surgical delay of 3 months." Cases that are recommended to be deferred include solid nodules or lung cancer under $2 \mathrm{~cm}$, predominantly ground glass nodules or cancers, and tumors with indolent histology (3).

Based upon the accumulated evidence, diagnosed, or suspected stage I and II lung cancer should be resected as soon as possible, and no later than 6 to 8 weeks after diagnosis depending on local conditions. Moderate evidence exists that time to surgery beyond this time increases mortality, with mixed evidence for effect on pathologic upstaging. Importantly, there is no evidence to support a distinction of $2 \mathrm{~cm}$ for whether resection of lung cancer should be performed immediately or may be delayed 3 months, as suggested in the ACS guidelines and the Thoracic Surgery Outcomes Research Network guidelines. In the one study specifically evaluating stage Ia lung cancer, a delay over 38 days was associated with worse mortality (17). Even subcentimeter lung cancers are at $10 \%$ risk for nodal involvement when solid, highlighting the importance of early resection (43). For stage IIIa lung cancer, evidence consistently shows delayed resection following neoadjuvant therapy is associated with lower survival. Of the included studies, the earliest delay associated with worse survival was greater than 6 weeks (9). These findings support timely resection as stated in the guidelines.

Suspected tumors with low malignant potential, such as suspected adenocarcinoma-in-situ or minimally-invasive adenocarcinoma, could likely be safely deferred with imaging to reassess for progression at three to 6-month intervals, in agreement with the aforementioned guidelines (44). Tumors with ground-glass opacities may also be safely delayed with serial imaging due to their improved prognosis and less aggressive nature. Imaging characteristics—such as consolidation relative to tumor diameter (CTR)—should be used to stratify the risk of aggressive disease and direct clinical decision making in ground-glass opacities on an individualized basis. In a retrospective cohort study, patients with ground-glass opacities with a CTR of 0 had a $4 \%$ risk of aggressive cancer, compared to patients with a CTR $>25 \%$ who had a $70 \%$ risk of aggressive cancer (45).

\section{Esophageal cancer}

During the COVID-19 pandemic, the ACS and Thoracic Surgery Research Outcomes Network recommend immediate surgery for esophageal cancer stage Ib or greater and esophageal cancer post neoadjuvant therapy, while suggesting endoscopic therapy for amenable early stage $\mathrm{Ia} / \mathrm{b}$ cancers (3). Esophageal cancer represents an aggressive malignancy and resection typically follows neoadjuvant therapy except in the earliest stage. Fittingly, most of the existing literature evaluates time to surgery following neoadjuvant therapy. In early esophageal cancer, minimal evidence exists regarding the impact of surgical delay on outcomes. The existing evidence suggests that prolonged time to surgery over 8 weeks may results in decreased survival, thus resection should not be delayed when possible.

The data for time to surgery following neoadjuvant therapy are mixed. A recent meta-analysis evaluating optimal time to esophagectomy following neoadjuvant chemoradiation demonstrated that time to surgery greater than seven to 8 weeks improved pathologic complete response (RR 1.13, 95\% CI: 1.05-1.21) but decreased overall survival (RR 0.88, 95\% CI: 0.82-0.95) (46). Our findings agree with this analysis: some evidence suggests a time to surgery of greater than 6 to 9 weeks following neoadjuvant therapy is associated with lower 
survival; however, greater delay improves the likelihood of pathologic complete response. Therefore, the optimal window for surgery after neoadjuvant therapy seems to be approximately 6 to 8 weeks. An important consideration during the COVID-19 pandemic is that there is some evidence of potential equivalence of definitive chemoradiation to trimodality therapy for overall survival, although salvage esophagectomy has increased complication risk $(47,48)$. In the setting of severe resource strain this should be considered.

\section{Considerations during the COVID-19 pandemic}

The COVID-19 pandemic presents unique challenges for triage of lung and esophageal surgery. The oncologic considerations-how quickly should we operate before progression and decreased survival-must be weighed among other considerations. Recently published literature investigating outcomes of perioperative COVID-19 infection in an international multicenter cohort study of 1,128 patients demonstrated $23.8 \% 30$-day mortality with $51.2 \%$ experiencing pulmonary complications (49). Importantly, COVID-19 infection was only diagnosed preoperatively in $26.1 \%$ of patients likely due to the large portion of emergency surgery in this group, although early infection or nosocomial infection postoperatively may also contribute. While many patients underwent emergency surgery and there was no control group, these findings are clearly concerning for high perioperative risk. Thoracic surgery patients undergoing either pulmonary or esophageal resection, who may already be deconditioned from neoadjuvant therapy or from malignancy, have little physiologic reserve for pulmonary complications and COVID-19 infection perioperatively would be devastating. In addition to routine preoperative COVID-19 screening, hospitals must implement strict infection control procedures to minimize the risk of nosocomial infections in this highrisk population.

While some recommendations for managing cancer during the COVID-19 pandemic include consideration of neoadjuvant therapy (instead of primary resection) or extension of neoadjuvant therapy (to delay surgery), this is not without additional risks that are difficult to quantify. In the setting of an overwhelmed hospital system with a high COVID-19 burden, neoadjuvant therapy may allow surgical resection to be delayed with decreased oncologic risk (i.e., the risk of cancer progression or decreased cancer-specific survival) and decreased risk of nosocomial infection in the perioperative period. However, immunosuppression during a pandemic carries the additional risk of infection or severe COVID-19 illness if contracted and neoadjuvant therapy still requires frequent interactions with the healthcare system.

Additional considerations include system-based factors involving the allocation of limited resources. The backlog of delayed surgical procedures is expected to last at least months with a return to normal volume, although additional surges of COVID-19 will continue to impact available resources on a local and regional level. Other high-acuity cancer operations, as well as urgent procedures for benign disease, will compete for scheduling. Furthermore, the risk to the workforce should be minimized. Joint guidelines from the ACS, the American Society of Anesthesiologists, the Association of perioperative Registered Nurses, and the American Hospital Association for reopening provide a roadmap involving assessment of local conditions, COVID-19 testing availability, personal protective equipment availability, considerations for case prioritization, perioperative considerations, and risk-mitigation (50).

The ongoing TERAVOLT study provides the first data regarding outcomes of COVID-19 infection in patients with thoracic malignancies (51). Two hundred consecutive patients presented across 42 institutions in eight countries from March 26, 2020 to April 12, 2020. One-third of these patients died and over one-half (53\%) required hospitalization over 8 days. While this is a startlingly high mortality rate-suggesting very poor outcomes for COVID-19 infection in patients with thoracic malignancyseveral limitations temper these conclusions and applicability to decision making in a surgically-resectable disease cohort: (I) very limited critical care was provided as only $6 \%$ of patients received mechanical ventilation and $9 \%$ were admitted to the ICU; (II) $74 \%$ of patients had stage IV disease; (III) $59 \%$ of patients were enrolled from Italy at the time of peak incidence with severe resource limitations; and (VI) patients were enrolled early in the global pandemic prior to established treatment protocols. Further results from this ongoing study are necessary to make broader conclusions about care of patients with resectable thoracic malignancies during the pandemic.

\section{Limitations}

First, the innate limitation of this study is defining the "acceptable" time to surgery and what appropriate outcomes should be considered. When a malignancy is 
discovered at an early stage prior to spread, timely surgical resection is often curative. No plausible mechanism would suggest a delayed time to surgery would be beneficial in the absence of other treatment; thus, the goal is to operate prior to progression. However, although cancer is a progressive disease, the progression is not linear. It starts with local invasion and growth prior to spreading elsewhere-to lymph nodes, adjacent organs, or to distant locations. Some cancers, such as prostate cancer and thyroid micropapillary carcinoma, are well known to remain at early stages for years without progression. Other cancers progress rapidly from early stages, such as esophageal and pancreatic cancer. Therefore, while the ideal time to cancer surgery is "without delay", the impact of operating within a certain timeframe likely has a greater impact for more aggressive cancers. Importantly, in the neoadjuvant setting, a longer delay to surgery may allow for improved pathologic response and potentially improved survival.

Second, most studies look at overall survival, diseasefree survival, or pathologic staging (either upstaging in the primary setting or downstaging after neoadjuvant therapy). Third, the included literature almost entirely consists of retrospective cohort studies of varying quality, with a combination of large database studies and small institutional studies. Time to surgery cutoffs were chosen arbitrarily in most studies based upon the patient population studied and varied dramatically between studies. Given the significant heterogeneity among patient populations and time to surgery cutoffs, the data could not be meaningfully grouped into a meta-analysis. Fourth, the reasons for delay are not investigated in almost all included studies. If comorbidities or other clinical problems requiring optimization were the source for the delay this may not be appropriately adjusted for. Also, socioeconomic, and other factors that contribute to worse outcomes may also contribute to a longer time to surgery. For every type of cancer, surgeons consistently operate more quickly for more advanced cancers in sicker patients. Even with careful adjustment, it is likely that studies are unable to account for all confounders given their mostly retrospective nature. Fifth, patients with longer surgical delays who progress to unresectable disease may not be accounted for in the studied patient populations. Sixth, our specific search methodology may not have identified all relevant literature as we only evaluated one database, although we did perform a careful citation review of included papers to identify additional relevant literature.

\section{Conclusions}

In the setting of the COVID-19 pandemic and unprecedented hospital strain, prioritization of scarce resources becomes critical. While surgical capacity starts to recover in the United States, the accumulated backlog of surgical disease and an impending "second wave" will require ongoing prioritization of operations for the months to come. For patients with operable cancer, the clock is ticking: cancer continues to grow and spread, even in a pandemic. While triage guidelines have been released, no source has previously compiled the available literature on the impact of time to surgery for these malignancies. Moderate evidence demonstrates worsened survival with delayed resection of lung cancer, even in early lung cancer. No evidence justifies a distinction in management of tumors greater than or less than $2 \mathrm{~cm}$. There does not appear to be a "safe delay" for lung cancer and these tumors should be resected promptly. In esophageal cancer, the optimal window for post-neoadjuvant resection appears to be 6 to 8 weeks. Minimal evidence suggests that primary esophagectomy should occur within 8 weeks.

In "Phase I," where hospital resources are largely preserved, resection of lung and esophageal cancer should not be delayed. Once resources are under greater strain, surgeons need to make case-by-case determinations to evaluate the risk of surgical delay, available hospital resources, and the risk to patients from potential nosocomial exposures to determine optimal management. Further, they should consider whether alternative therapies, such as neoadjuvant therapy or endoscopic therapy, might temporize disease progression and extend the safe window to surgery. Ultimately, it is critical that decisions on whether to delay surgery are based upon the available evidence.

\section{Acknowledgments}

Funding: None.

\section{Footnote}

Reporting Checklist: The authors have completed the PRISMA reporting checklist. Available at http://dx.doi. org/10.21037/jtd-20-2400

Conflicts of Interest: All authors have completed the ICMJE uniform disclosure form (available at http://dx.doi. 
org/10.21037/jtd-20-2400). SPG serves as an unpaid editorial board member of Fournal of Thoracic Disease from May 2019 to Apr 2021. The authors have no other conflicts of interest to declare.

Ethical Statement: The authors are accountable for all aspects of the work in ensuring that questions related to the accuracy or integrity of any part of the work are appropriately investigated and resolved. All data extracted from published manuscripts; ethical review and informed consent not applicable.

Open Access Statement: This is an Open Access article distributed in accordance with the Creative Commons Attribution-NonCommercial-NoDerivs 4.0 International License (CC BY-NC-ND 4.0), which permits the noncommercial replication and distribution of the article with the strict proviso that no changes or edits are made and the original work is properly cited (including links to both the formal publication through the relevant DOI and the license). See: https://creativecommons.org/licenses/by-nc-nd/4.0/.

\section{References}

1. Centers for Disease Control and Prevention. Interim Guidance for Healthcare Facilities: Preparing for Community Transmission of COVID-19 in the United States 2020. Available online: https://www.cdc.gov/ coronavirus/2019-ncov/healthcare-facilities/guidancehcf.html

2. American College of Surgeons. COVID-19: Guidance for Triage of Non-Emergent Surgical Procedures 2020. Available online: https://www.facs.org/about-acs/covid-19/ information-for-surgeons/triage

3. Thoracic Surgery Outcomes Research Network, Inc, Antonoff M, Backhus L, et al. COVID-19 Guidance for Triage of Operations for Thoracic Malignancies: A Consensus Statement from Thoracic Surgery Outcomes Research Network. Ann Thorac Surg 2020;110:692-6.

4. Liberati A, Altman DG, Tetzlaff J, et al. The PRISMA statement for reporting systematic reviews and metaanalyses of studies that evaluate health care interventions: explanation and elaboration. Ann Intern Med 2009;151:W65-94.

5. Phillips B, Ball, B, Sackett, D, et al. Oxford Centre for Evidence-based Medicine - Levels of Evidence 2009. Available online: https://www.cebm.net/2009/06/oxfordcentre-evidence-based-medicine-levels-evidence- march-2009/

6. Wells G SB, O'Connell D, Peterson J, et al. The Newcastle-Ottawa Scale (NOS) for assessing the quality of nonrandomised studies in meta-analyses 2003. Available online: http://www.lri.ca/ programs/ceu/oxford.htm

7. Bott MJ, Patel AP, Crabtree TD, et al. Pathologic Upstaging in Patients Undergoing Resection for Stage I Non-Small Cell Lung Cancer: Are There Modifiable Predictors? Ann Thorac Surg 2015;100:2048-53.

8. Coughlin S, Plourde M, Guidolin K, et al. Is it safe to wait? The effect of surgical wait time on survival in patients with non-small cell lung cancer. Can J Surg 2015;58:414-8.

9. Gao SJ, Corso CD, Wang EH, et al. Timing of Surgery after Neoadjuvant Chemoradiation in Locally Advanced Non-Small Cell Lung Cancer. J Thorac Oncol 2017;12:314-22.

10. Kanarek NF, Hooker CM, Mathieu L, et al. Survival after community diagnosis of early-stage non-small cell lung cancer. Am J Med 2014;127:443-9.

11. Liberman M, Liberman D, Sampalis JS, et al. Delays to surgery in non-small-cell lung cancer. Can J Surg 2006;49:31-6.

12. Marulli G, Verderi E, Comacchio GM, et al. Predictors of unexpected nodal upstaging in patients with cT1$3 \mathrm{~N} 0$ non-small cell lung cancer (NSCLC) submitted to thoracoscopic lobectomy. J Vis Surg 2018;4:15.

13. Odell DD, Feinglass J, Engelhardt K, et al. Evaluation of adherence to the Commission on Cancer lung cancer quality measures. J Thorac Cardiovasc Surg 2019;157:1219-35.

14. Rice JD, Heidel J, Trivedi JR, van Berkel VH. Optimal Surgical Timing After Neoadjuvant Therapy for Stage IIIa Non-Small Cell Lung Cancer. Ann Thorac Surg 2020;109:842-7.

15. Samson P, Patel A, Garrett T, et al. Effects of Delayed Surgical Resection on Short-Term and Long-Term Outcomes in Clinical Stage I Non-Small Cell Lung Cancer. Ann Thorac Surg 2015;99:190612; discussion 1913.

16. Shin DW, Cho J, Kim SY, et al. Delay to curative surgery greater than 12 weeks is associated with increased mortality in patients with colorectal and breast cancer but not lung or thyroid cancer. Ann Surg Oncol 2013;20:2468-76.

17. Yang CJ, Wang H, Kumar A, et al. Impact of Timing of Lobectomy on Survival for Clinical Stage IA Lung Squamous Cell Carcinoma. Chest 2017;152:1239-50. 
18. Chiu CH, Chao YK, Chang HK, et al. Interval between neoadjuvant chemoradiotherapy and surgery for esophageal squamous cell carcinoma: does delayed surgery impact outcome? Ann Surg Oncol 2013;20:4245-51.

19. Franko J, Voynov G, Goldman CD. Esophagectomy Timing After Neoadjuvant Therapy for Distal Esophageal Adenocarcinoma. Ann Thorac Surg 2016;101:1123-30.

20. Franko J, McAvoy S. Timing of esophagectomy after neoadjuvant chemoradiation treatment in squamous cell carcinoma. Surgery 2018;164:455-9.

21. Furukawa T, Hamai Y, Hihara J, et al. Impact of Interval Between Neoadjuvant Chemoradiation and Surgery Upon Morbidity and Survival of Patients with Squamous Cell Carcinoma of Thoracic Esophagus. Anticancer Res 2018;38:5239-45.

22. Grotenhuis BA, van Hagen P, Wijnhoven BP, et al. Delay in diagnostic workup and treatment of esophageal cancer. J Gastrointest Surg 2010;14:476-83.

23. Haisley KR, Laird AE, Nabavizadeh N, et al. Association of Intervals Between Neoadjuvant Chemoradiation and Surgical Resection With Pathologic Complete Response and Survival in Patients With Esophageal Cancer. JAMA Surg 2016;151:e162743.

24. Kathiravetpillai N, Koeter M, van der Sangen MJ, et al. Delaying surgery after neoadjuvant chemoradiotherapy does not significantly influence postoperative morbidity or oncological outcome in patients with oesophageal adenocarcinoma. Eur J Surg Oncol 2016;42:1183-90.

25. Kim JY, Correa AM, Vaporciyan AA, et al. Does the timing of esophagectomy after chemoradiation affect outcome? Ann Thorac Surg 2012;93:207-12; discussion 212-3.

26. Klevebro F, Nilsson K, Lindblad M, et al. Association between time interval from neoadjuvant chemoradiotherapy to surgery and complete histological tumor response in esophageal and gastroesophageal junction cancer: a national cohort study. Dis Esophagus 2020;33:doz078.

27. Lee A, Wong AT, Schwartz D, et al. Is There a Benefit to Prolonging the Interval Between Neoadjuvant Chemoradiation and Esophagectomy in Esophageal Cancer? Ann Thorac Surg 2016;102:433-8.

28. Raman V, Jawitz OK, Voigt SL, et al. Effect of time to surgery on outcomes in stage I esophageal adenocarcinoma. J Thorac Cardiovasc Surg 2020;159:1626-35.e1.

29. Ranney DN, Mulvihill MS, Yerokun BA, et al. Surgical resection after neoadjuvant chemoradiation for oesophageal adenocarcinoma: what is the optimal timing? Eur J Cardiothorac Surg 2017;52:543-51.
30. Ruol A, Rizzetto C, Castoro C, et al. Interval between neoadjuvant chemoradiotherapy and surgery for squamous cell carcinoma of the thoracic esophagus: does delayed surgery have an impact on outcome? Ann Surg 2010;252:788-96.

31. Shaikh T, Ruth K, Scott WJ, et al. Increased time from neoadjuvant chemoradiation to surgery is associated with higher pathologic complete response rates in esophageal cancer. Ann Thorac Surg 2015;99:270-6.

32. Shapiro J, van Hagen P, Lingsma HF, et al. Prolonged time to surgery after neoadjuvant chemoradiotherapy increases histopathological response without affecting survival in patients with esophageal or junctional cancer. Ann Surg 2014;260:807-13; discussion 813-4.

33. Singla S, Gabriel E, Alnaji R, et al. Complete pathologic response is independent of the timing of esophagectomy following neoadjuvant chemoradiation for esophageal cancer. J Gastrointest Oncol 2018;9:73-9.

34. Tessier W, Gronnier C, Messager M, et al. Does timing of surgical procedure after neoadjuvant chemoradiation affect outcomes in esophageal cancer? Ann Thorac Surg 2014;97:1181-9.

35. Tsang JS, Tong DKH, Lam KO, et al. Appropriate timing for surgery after neoadjuvant chemoradiation for esophageal cancer. Dis Esophagus 2017;30:1-8.

36. van der Werf LR, Dikken JL, van der Willik EM, et al. Time interval between neoadjuvant chemoradiotherapy and surgery for oesophageal or junctional cancer: A nationwide study. Eur J Cancer 2018;91:76-85.

37. van der Wilk BJ, Noordman BJ, Neijenhuis LKA, et al. Active Surveillance Versus Immediate Surgery in Clinically Complete Responders After Neoadjuvant Chemoradiotherapy for Esophageal Cancer: A Multicenter Propensity Matched Study. Ann Surg 2019. [Epub ahead of print].

38. Visser E, Leeftink AG, van Rossum PS, et al. Waiting Time from Diagnosis to Treatment has no Impact on Survival in Patients with Esophageal Cancer. Ann Surg Oncol 2016;23:2679-89.

39. Visser E, van Rossum PS, Leeftink AG, et al. Impact of diagnosis-to-treatment waiting time on survival in esophageal cancer patients - A population-based study in The Netherlands. Eur J Surg Oncol 2017;43:461-70.

40. Wang BY, Chen HS, Hsu PK, et al. Clinical impact of the interval between chemoradiotherapy and esophagectomy in esophageal squamous cell carcinoma patients. Ann Thorac Surg 2015;99:947-55.

41. National Comprehensive Cancer Network. Non-Small 
Cell Lung Cancer (Version 3. 2020) 2020. Available online: https://www.nccn.org/professionals/physician_gls/pdf/ nscl_blocks.pdf

42. American College of Surgeons. CoC Quality of Care Measures 2016. Available online: https://www.facs.org/ quality-programs/cancer/ncdb/qualitymeasures

43. Sakurai H, Nakagawa K, Watanabe S, Asamura H. Clinicopathologic features of resected subcentimeter lung cancer. Ann Thorac Surg 2015;99:1731-8.

44. Kakinuma R, Noguchi M, Ashizawa K, et al. Natural History of Pulmonary Subsolid Nodules: A Prospective Multicenter Study. J Thorac Oncol 2016;11:1012-28.

45. Sawada S, Yamashita N, Sugimoto R, et al. Long-term Outcomes of Patients With Ground-Glass Opacities Detected Using CT Scanning. Chest 2017;151:308-15.

46. Qin Q, Xu H, Liu J, et al. Does timing of esophagectomy following neoadjuvant chemoradiation affect outcomes? A meta-analysis. Int J Surg 2018;59:11-8.

47. Voeten DM, den Bakker CM, Heineman DJ, et al. Definitive Chemoradiotherapy Versus Trimodality

Cite this article as: Fligor SC, Tsikis ST, Wang S, Ore AS, Allar BG, Whitlock AE, Calvillo-Ortiz R, Arndt K, Callery MP, Gangadharan SP. Time to surgery in thoracic cancers and prioritization during COVID-19: a systematic review. J Thorac Dis 2020;12(11):6640-6654. doi: 10.21037/jtd-20-2400
Therapy for Resectable Oesophageal Carcinoma: Metaanalyses and Systematic Review of Literature. World J Surg 2019;43:1271-85.

48. Jamel S, Markar SR. Salvage esophagectomy: safe therapeutic strategy? J Thorac Dis 2017;9:S799-808.

49. COVIDSurg Collaborative. Mortality and pulmonary complications in patients undergoing surgery with perioperative SARS-CoV-2 infection: an international cohort study. Lancet 2020;396:27-38.

50. American College of Surgeons, American Society of Anesthesiologists, Association of periOperative Registered Nurses, American Hospital Association. Joint Statement: Roadmap for Resuming Elective Surgery after COVID-19 Pandemic 2020. Available online: https://www.facs.org/ covid-19/clinical-guidance/roadmap-elective-surgery

51. Garassino MC, Whisenant JG, Huang LC, et al. COVID-19 in patients with thoracic malignancies (TERAVOLT): first results of an international, registrybased, cohort study. Lancet Oncol 2020;21:914-22. 